\title{
Optic perineuritis: a retrospective case series
}

This article was published in the following Dove Press journal:

International Medical Case Reports Journal

8 June 2017

Number of times this article has been viewed

\section{Olle Bergman' \\ Tommy Andersson' \\ Madeleine Zetterberg ${ }^{1,2}$ \\ 'Department of Ophthalmology, Sahlgrenska University Hospital, \\ ${ }^{2}$ Department of Clinical \\ Neuroscience/Ophthalmology, \\ Institute of Neuroscience and \\ Physiology, University of Gothenburg, \\ Mölndal, Sweden}

Correspondence: Olle Bergman Department of Ophthalmology, Sahlgrenska University Hospital, SE 43I 80 Mölndal, Sweden

$\mathrm{Tel}+4670921096 \mathrm{I}$

Email olle.bergman@vgregion.se
Abstract: Optic perineuritis (OPN) is a rare inflammatory disorder involving the optic nerve sheath characterized by visual loss. OPN may be isolated and idiopathic or part of an underlying disorder. This case series aimed to help clinicians investigate and manage this disorder. Presentation, clinical findings, and treatment of OPN are discussed. After review of medical records at the ophthalmology clinic at Sahlgrenska University hospital in Gothenburg, Sweden, seven OPN patients (three men and four women) were identified and included in the present case series. These included idiopathic cases and patients with underlying disorders. Age at OPN diagnosis ranged from 26 to 64 years (mean age 55 years, median age 58 years). Five of the patients were treated with corticosteroids. This study suggests that a high-dose course of corticosteroids is important in the treatment of OPN in severely affected patients.

Keywords: glucocorticoids, prednisone, vision, corticosteroids, inflammation, idiopathic, magnetic resonance imaging

\section{Introduction}

Optic perineuritis (OPN) is a rare inflammatory disorder involving the optic nerve sheath characterized by visual loss. Although many cases of OPN are understood to be isolated and idiopathic, ${ }^{1-4}$ patients with OPN secondary to inflammatory or infectious disorders, including syphilis, ${ }^{5,6}$ sarcoidosis, ${ }^{7,8}$ Crohn disease, ${ }^{9}$ and Wegener's granulomatosis, ${ }^{3}$ have also been reported. Given the various underlying disorders associated with the disorder, OPN is conceivably several separate disorders that share the feature of optic nerve sheath inflammation. Treatment of OPN typically requires high doses of corticosteroids, but relapse following discontinued treatment is common, ${ }^{2,10}$ although spontaneous resolution has been reported. ${ }^{11}$

Clinically, OPN may present itself similarly to acute optic neuritis (ON). Both the disorders affect the optic nerve. Moreover, both are more common in women and typically involve acute monocular visual loss, eye movement-related pain, and disc edema. ${ }^{2,12}$ Diagnosis of OPN is consequently usually dependent on radiographic features. Since computed tomographic scanning usually does not provide enough spatial resolution to differentiate OPN from $\mathrm{ON},{ }^{2} \mathrm{OPN}$ is best visualized by using fat-suppressed, contrasted coronal functional magnetic resonance imaging (MRI) sequences. ${ }^{13}$ Findings typically consist of contrast enhancement surrounding the intra-orbital optic nerve with sparing of the nerve itself. ${ }^{14}$ The distinction is important since the prognosis differs between the two; patients with ON are more likely to develop multiple sclerosis (MS), whereas OPN patients are not. Moreover, response to treatment with corticosteroids is usually 
prompt and dramatic in patients with OPN, whereas response in patients with $\mathrm{ON}$ is more variable. ${ }^{2}$ Moreover, a previous study by Purvin et al showed that the visual loss experienced by patients with OPN usually takes place over several weeks, whereas in ON it occurs more rapidly, usually over some days. Sparing of central vision was found to be more common in patients with OPN than those with ON. ${ }^{2}$

In this retrospective case series, the medical records and imaging studies of seven OPN patients treated at a tertiary ophthalmology clinic were reviewed in order to better characterize the clinical features of OPN.

\section{Methods}

A total of 627 patients treated at the ophthalmology clinic at Sahlgrenska University hospital in Gothenburg, Sweden, were identified, whose medical records included the ICD-10 codes: H46.9 (unspecified ON), H47.0 (disorders of optic nerve, not elsewhere classified), H47.1 (papilledema), H47.3 (other disorders of optic disc), H48.1 (retrobulbar neuritis in diseases classified elsewhere), and H48.8 (other disorders of optic nerve and visual pathways in diseases classified elsewhere). After review of the medical records, seven OPN patients were identified and described in this communication.

Inclusion criteria included contrast enhancement surrounding the intra-orbital optic nerve, and at least one of the following clinical symptoms: 1) reduction of visual acuity, 2) impairment of visual field, and 3) eye pain.

Symptoms, visual acuity, color vision, visual field, disc appearance, and imaging findings were reviewed, as was treatment and response. Best-corrected visual acuity (BCVA) was measured monocularly by using Snellen charts.

Presence of systemic disorders was not considered an exclusionary factor. The follow-up time for included patients was 2-24 months.

The ethics committee at the University of Gothenburg approved this study. Patient written informed consent to publish this paper and accompanying images was obtained.

\section{Case presentations}

The OPN patients included three men and four women. Age at OPN diagnosis ranged from 26 to 64 years (mean age 55 years, median age 58 years). Five of the patients were treated with corticosteroids. The cases are summarized in Table 1.

\section{Patient I}

A 60-year-old man developed pain in the left eye 3 days prior to his first visit. At the ophthalmological consultation, he negated any previous disorders, except a history of migraine, and was not taking any prescription medication. The patient displayed a slight exophthalmos of the left eye. BCVA was 0.8 in the right eye and 1.0 in his left eye. A fundus examination revealed a normal optic disc and retina in both eyes. The patient did not receive any treatment except tear substitution eye drops. Laboratory testing included determination of hemoglobin (HGB), glucose, C-reactive protein (CRP), thyroid-stimulating hormone (TSH), thyroxine (T4), and erythrocyte sedimentation rate (ESR), and results from all tests were normal. An orbital MRI with and without intravenous contrast carried out 3 weeks later revealed moderately increased signal intensity in the optical nerve sheaths, especially on the left side, in line with what is seen in OPN. The MRI also showed increased signaling in the retrobulbar fat. No swelling of the extraocular muscles was evident.

The eye pain had subsided at a new visit 2 months after the initial consultation. Visual acuity was measured 2, 5, and 12 months after the first visit; corrected visual acuity was 1.0 in both eyes on these examinations. Visual field and color vision tests were normal on all three follow-up examinations. Fundus examinations were performed on all three visits and did not reveal anything abnormal.

\section{Patient 2}

A 60-year-old male experienced dimmed vision in his left eye for 3 days prior to his first visit. The patient negated any other symptoms, including pain and diplopia. He had a history of two previous occurrences of possible $\mathrm{ON}-30$ years ago in his left eye and some 10 years later in his right eye. The patient had a history of hypertension and was currently on antihypertensive drugs. No relative afferent pupillary defect (RAPD) was present at his first examination. BCVA was 0.65 in the right eye and 0.5 in his left eye. A fundus examination revealed an atrophic and excavated disc in his right eye. An optic disc edema was present in his left eye. Retina was normal in both eyes. Laboratory tests for sarcoidosis, syphilis, and giant cell arteritis were analyzed, including determination of HGB, blood count, serum calcium, ESR, angiotensin-converting enzyme (ACE), Venereal Disease Research Laboratory (VDRL), and Wassermann reaction (WR). All tests were normal or negative. Visual field and color vision were analyzed 3 days later; visual field test showed arcuate inferior defects in both eyes, with equivalent neural capacity of $67 \%$ in his right eye and 58\% in his left eye. A Sahlgren's saturation test (SST) color vision test revealed a more defective color vision in his left (20) than in his right (15) eye.

An orbital MRI carried out 1 week later revealed increased signal intensity in the parts of the left optical nerve sheath, 
Table I Clinical features at first examination and visual acuity at first and last examination

\begin{tabular}{|c|c|c|c|c|c|c|c|c|c|}
\hline ID & $\begin{array}{l}\text { Age } \\
\text { and sex }\end{array}$ & $\begin{array}{l}\text { BCVA at } \\
\text { first visit }\end{array}$ & $\begin{array}{l}\text { Visual field } \\
\text { defect }\end{array}$ & $\begin{array}{l}\text { Optic disc } \\
\text { appearance }\end{array}$ & $\begin{array}{l}\text { Associated } \\
\text { features } \\
\text { and initial } \\
\text { symptoms }\end{array}$ & Treatment & $\begin{array}{l}\text { Follow-up time } \\
\text { relapses }\end{array}$ & $\begin{array}{l}\text { e/BCVA } \\
\text { last visit }\end{array}$ & MRI findings \\
\hline Patient I & $\begin{array}{l}60 \text { years, } \\
\text { male }\end{array}$ & $0.8 / 1.0$ & Normal & Normal & $\begin{array}{l}\text { Left eye: pain, } \\
\text { exophthalmus }\end{array}$ & None & 12 months/none & $1.0 / 1.0$ & $\begin{array}{l}\text { Signal increase in optic } \\
\text { nerve sheath left eye }\end{array}$ \\
\hline Patient 2 & $\begin{array}{l}60 \text { years, } \\
\text { male }\end{array}$ & $0.65 / 0.5$ & $\begin{array}{l}\text { Bilateral } \\
\text { arcuate } \\
\text { inferior and } \\
\text { temporal } \\
\text { defect }\end{array}$ & Edema & $\begin{array}{l}\text { Left eye: dimmed } \\
\text { vision }\end{array}$ & Prednisolone & 8 months/none & I.0/0.5 & $\begin{array}{l}\text { Inflammation in orbita } \\
\text { fat and nerve sheaths } \\
\text { bilaterally }\end{array}$ \\
\hline Patient 3 & $\begin{array}{l}58 \text { years, } \\
\text { female }\end{array}$ & $\mathrm{HM} / \mathrm{I} .0$ & Not stated & Normal & $\begin{array}{l}\text { Right eye: } \\
\text { dimmed vision }\end{array}$ & Prednisolone & 18 months/none & $0.5 / 1.0$ & $\begin{array}{l}\text { Optic nerve sheath } \\
\text { enhancement }\end{array}$ \\
\hline Patient 4 & $\begin{array}{l}26 \text { years, } \\
\text { female }\end{array}$ & $1.3 / 1.0$ & $\begin{array}{l}\text { Inferior } \\
\text { temporal } \\
\text { defect left eye }\end{array}$ & Normal & $\begin{array}{l}\text { Left eye: } \\
\text { dimmed vision, } \\
\text { movement- } \\
\text { associated pain }\end{array}$ & $\begin{array}{l}\text { IVMP and } \\
\text { prednisolone }\end{array}$ & $\begin{array}{l}7 \text { months/I } \\
\text { relapse }\end{array}$ & $1.3 / 1.6$ & $\begin{array}{l}\text { Increased signal } \\
\text { intensity in the optical } \\
\text { nerve sheaths of both } \\
\text { eyes }\end{array}$ \\
\hline Patient 5 & $\begin{array}{l}58 \text { years, } \\
\text { male }\end{array}$ & I.0/0.1 & Not stated & Edema & $\begin{array}{l}\text { Left eye: dimmed } \\
\text { vision, RAPD, } \\
\text { color vision } \\
\text { defect, headache }\end{array}$ & None & 2 months/none & $0.8 / 1.0$ & $\begin{array}{l}\text { Slight increase in signal } \\
\text { intensity in the optic } \\
\text { nerve sheath of left } \\
\text { eye }\end{array}$ \\
\hline Patient 6 & $\begin{array}{l}64 \text { years, } \\
\text { female }\end{array}$ & $0.8 / 0.8$ & $\begin{array}{l}\text { Temporal } \\
\text { defect in both } \\
\text { eyes }\end{array}$ & $\begin{array}{l}\text { Peripapillary } \\
\text { atrophy. No } \\
\text { edema }\end{array}$ & $\begin{array}{l}\text { SLE. Gradual } \\
\text { visual loss } \\
\text { bilaterally over } \\
\text { several months }\end{array}$ & $\begin{array}{l}\text { IVMP and } \\
\text { prednisolone }\end{array}$ & 10 months/none & $0.8 / 0.65$ & $\begin{array}{l}\text { Increased signal } \\
\text { intensity in the optical } \\
\text { nerve sheaths }\end{array}$ \\
\hline Patient 7 & $\begin{array}{l}56 \text { years, } \\
\text { female }\end{array}$ & $0.8 / 0.13$ & Not stated & Edema & $\begin{array}{l}\text { Left eye: RAPD, } \\
\text { pain, gradual } \\
\text { vision loss } \\
\text { over } 2 \text { months. } \\
\text { Sarcoidosis } \\
\text { confirmed }\end{array}$ & $\begin{array}{l}\text { Prednisolone, } \\
\text { azathioprine }\end{array}$ & $\begin{array}{l}24 \text { months/ } \\
3 \text { relapses }\end{array}$ & $0.5 / 0.65$ & $\begin{array}{l}\text { Enhancement in the } \\
\text { left nerve sheath }\end{array}$ \\
\hline
\end{tabular}

Abbreviations: BCVA, best-corrected visual acuity; HM, hand movement perception; ID, identification; IVMP, intravenous methylprednisolone; SLE, systemic lupus erythematosus; RAPD, relative afferent pupillary defect; MRI, magnetic resonance imaging.

possibly indicating OPN. The MRI also showed increased signaling in the retrobulbar fat of the left eye. No increased swelling of the extraocular muscles was evident.

The patient received a course of prednisone, $60 \mathrm{mg} /$ day, which was tapered over 1 month, at which time he was examined again. BCVA was 1.0 in the right eye and 0.65 in his left eye. The optic disc edema had subsided substantially since the last fundus examination 1 month earlier. He received a new course of prednisone, starting with $25 \mathrm{mg} /$ day, which was tapered to $7.5 \mathrm{mg}$ /day until his next visit 2 months later. His BCVA was 1.0 in his right eye and 0.65 in his left eye.

Visual field testing again showed arcuate inferior defects in both eyes and abnormal thresholds, with equivalent neural capacity of $69 \%$ in his right eye and $61 \%$ in his left eye. A new MRI showed a slight regression of the enhancement around the left optical nerve since the last MRI. The results of a fundus examination revealed a pale papilla in both eyes. He remained on $7.5 \mathrm{mg}$ /day prednisone, which was tapered over 14 weeks. At a new consultation 4 months later, BCVA was 1.0 in the right eye and 0.5 in his left eye.

\section{Patient 3}

A 58-year-old woman experienced sudden deterioration of vision in her right eye, 2 weeks prior to her first visit. The patient negated any other symptoms related to her eyes, including pain and diplopia. The patient had a history of pain-related symptoms in her arms and negated taking any medicines regularly.

An initial eye examination determined BCVA to be 1.0 in her left eye, whereas she was only able to distinguish hand movement in her right eye. No RAPD was evident at this first examination. No proptosis or enophtalmus was present. A fundus examination revealed that both eyes had vital optic discs and normal retinas. Initial laboratory tests revealed a high ESR ( $22 \mathrm{~mm} / \mathrm{h})$, a slightly increased CRP ( $8 \mathrm{mg} / \mathrm{L})$, and normal HGB and creatinine. The patient negated head pain, scalp tenderness, and jaw pain.

An orbital MRI was carried out 3 weeks after her initial visit and showed a suspected increase in fluid around the distal part of the optical nerve of the right eye, along with increased signaling. The pituitary gland also displayed 
increased signaling. The results were interpreted as being suggestive of OPN. The patient received a course of prednisone, starting with $60 \mathrm{mg} /$ day for 5 days, $50 \mathrm{mg} /$ day for 10 days, and $40 \mathrm{mg} /$ day until her next appointment 3 months after her initial examination.

At the follow-up, an examination determined BCVA to be 1.3 in her left eye and $<0.5$ in her right eye. Because of severe side effects, the prednisone was tapered to $5 \mathrm{mg}$ /day at her next visit 6 weeks later. BCVA was determined to be 1.0 in her left eye and 0.5 in her right eye. Color vision was assessed and found to be defective in her right eye (red light seen as pink). Prednisone was tapered out completely during the next 10 days. A new MRI showed increased signaling in the right optic nerve and right nerve sheath.

At a new examination 4 months later (8 months after her first visit), BCVA was unchanged. A visual field test of her right eye revealed a lower arcuate scotoma (Humphrey field test, visual field index [VFI] of 94\%). Fundus examination revealed a pale papilla in her right eye compared to the left. The macula appeared to be normal.

A MRI of the sella turcica area and a chest x-ray was conducted. No pathological changes were observed. Further laboratory tests included markers for sarcoidosis (ACE), syphilis (WR, VDRL), and inflammation. Besides a slightly elevated haptoglobin, results did not support any increase in inflammatory activity.

Her symptoms were unchanged at her next ophthalmologic examination 10 months later (one and a half years after her first visit), once again showing a paler papilla in her right eye compared to her left. A RAPD was present in her right eye.

At her last eye examination, 18 months after the initial contact, the optic disc in her right eye was found to be pale and slightly atrophic compared to the left eye. BCVA was unchanged at 1.0 in her left eye and 0.5 in her right eye. RAPD was present.

\section{Patient 4}

A 26-year-old woman presented with a 2-week history of cold-like symptoms prior to her first visit. She described having had 5 days of movement-associated eye pain and 3 days of dimmed vision in her left peripheral visual field. The patient also stated having a headache. No previous history of eye disorders was acknowledged. She was taking antibiotics (phenoxymethylpenicillin and chloramphenicol eye drops) for her symptoms.

No RAPD was present at the initial examination. BCVA was 1.3 in the right eye and $<1.0$ in her left eye. The results of a fundus examination showed a normal retina, papilla, and macula in both eyes. An SST color vision test did not reveal any abnormalities (0p). A confrontation visual field exam revealed a possible inferior temporal defect in the left eye. The overall lack of findings in the examination did not lend support for any medical treatment.

Two weeks later, her vision deteriorated during a trip abroad. She could only perceive hand waving on her right eye and finger counting on her left. An MRI scan of the spinal cord, brain, and orbita revealed increased signal intensity in the optical nerve sheaths of both eyes, suggesting OPN. There was no evidence of any other abnormalities in the brain or spinal cord. She was being treated with methylprednisone $1 \mathrm{~g}$ /day for 5 days and prednisone $50 \mathrm{mg}$ until her next examination 2 weeks later.

At this visit, the patient reported that her vision had normalized and that the pain had disappeared. BCVA was 1.3 in the right eye and 1.6 in her left eye. No RAPD was present. A visual field test did not demonstrate any irregularities on either eye (Humphrey field test, VFI was $100 \%$ in right eye and 98\% in left eye). An SST color vision test suggested slightly more defective color vision in her right (15) than in her left (10) eye. As was the case at her first visit, a fundus examination showed a normal retina, papilla, and macula in both eyes. Except a small bulge on her left iris, possibly an iris cyst, nothing abnormal was found. Her prednisone treatment was tapered out during a period of 1 month.

Five weeks later, the woman contacted the hospital and described similar symptoms as earlier, including a severe headache coupled with eye movement. Her steroid treatment had been completed 1 week earlier. No RAPD was present. A visual field test revealed a defect in the lower quadrants of her right eye (Humphrey field test, VFI was $96 \%$ in the right eye and $98 \%$ in the left eye). A fundus examination again showed a normal retina, macula, and papilla. Steroid treatment was reinstated with $40 \mathrm{mg}$ /day of prednisone.

Three weeks later, the patient was recuperating and reported that her eye sight had improved. A fundus examination revealed nothing abnormal. Her prednisone dose was slowly tapered. Seven months after her first visit, BCVA was 1.3 in her right eye and 1.6 in the left eye. An eye examination revealed nothing abnormal except for a persisting visual field defect in her left eye.

\section{Patient 5}

A 58-year-old male presented with a 2-day history of dimmed vision on his left eye. He reported having had headaches and swollenness behind his left eye during the last week. 
The patient had undergone heart surgery after a myocardial infarction 9 months prior to his first visit. He was taking several prescription medications because of hypertension and stent implants in his heart. These included aspirin, ticagrelor, bisoprolol, candesartan, and atorvastatin. Except for a pollen allergy, he negated other disorders.

An eye examination revealed free eye motility in all directions and slight movement-associated pain in his left eye. BCVA was 1.0 in the right eye and 0.1 in his left eye. There was no diplopia. A RAPD was present in his left eye at the same examination. Color vision was affected in the left eye. The results of a fundus examination revealed a normal optic disc in the right eye, except adjacent ectopic myelin in the lower portion. Retina and macula were without signs of disease. Examination of the left eye revealed papillary edema. Macula and retina were normal except for small hemorrhage from a vessel in the lower fundus. Blood pressure was 150/90. Initial laboratory tests that included ESR, CRP, serum calcium, ACE, anti-nuclear antibodies (ANAs), anti-neutrophil cytoplasmic antibodies (ANCA), and syphilis screen were normal, except for a slightly elevated IgA test.

An MRI of the orbita was performed a week after his first visit to the clinic and revealed a slight edema surrounding the left optical nerve, fitting with OPN. At this time, the patient reported that his vision had normalized. Steroid treatment was therefore not initiated. Since his first visit, he reported having three episodes of lost vision in his previously unaffected right eye a few days earlier. Also present was minor pain in his left eye and a few shorter episodes of blurred vision.

At a new visit, 3 weeks after his first, vision had improved further. A slight RAPD was present in his left eye. A fundus examination revealed no edema on either eye. Two months after his initial visit, BCVA was 0.8 in his right eye and 1.0 in the left eye. A visual field test conducted at the same time revealed a small isolated central scotoma with a slightly elevated threshold in his left eye (Humphrey field test, VFI was $100 \%$ in right eye and $99 \%$ in left eye).

\section{Patient 6}

A 64-year-old woman experienced gradual visual loss for several months prior to her first examination. The woman had a 15-year history of systemic lupus erythematosus (SLE). In addition, she suffered from hypertension, hyperlipidemia, chronic obstructive pulmonary disease (COPD), sleep apnea, and a sense of unsteadiness, which had worsened during the last months. She was on several medications (aspirin, omeprazole, simvastatin, atenolol, ramipril, amlodipine, albuterol inhalation, budenoside, and formoterol inhalation).
She negated eye pain. BCVA was 0.8 in both eyes. Both visual fields exhibited temporal defects (Humphrey field test). A fundus examination showed peripapillary atrophy in both eyes, but no sign of edema. An MRI with and without intravenous contrast performed 1 week later revealed an increased volume of fluid surrounding both optical nerves as well as increased signal intensity in the optical nerve sheaths, as seen in OPN. The MRI also showed increased signaling in the retrobulbar fat of the left eye and possible subcortical vasculitis. At a consultation, 1 week after the first visit, BCVA was unchanged since the last visit. Laboratory testing was done for sarcoidosis (ACE), syphilis (WR, VDRL), and neuromyelitis optica (NMO). In addition, ESR, CRP, HGB, and blood count were determined. Results showed a high or abnormal ESR, serum lysozyme, polyclonal IgG, and creatinine. Results for NMO antibodies, syphilis, and sarcoidosis markers were normal or negative. A chest X-ray did not find any sign of sarcoidosis. However, a slightly enlarged heart was observed, suggesting a developing heart failure. The woman was later administered intravenous corticosteroids $(1 \mathrm{~g} \times 2)$ and cyclophosphamide to treat her SLE. Shortly after, the woman was hospitalized due to cardiac arrhythmia. Warfarin and digoxin were prescribed, and the woman was discharged from the hospital some days later. Because of her arrhythmia, future treatment with corticosteroids was restricted.

At a new examination 3 months after her first visit, BCVA was unchanged. Results from eye field tests were still abnormal, with no certain change since the last examination. An SST color vision test showed more defective color vision in the left eye ( $80 \mathrm{p}$ ), compared to the right (30 p). Fundus examination showed no optic disc edema in either eye.

At a new consultation 2 months later, BCVA was still unchanged at 0.8 in both eyes. She was on prednisone (5mg/ day) in addition to methotrexate ( $5 \mathrm{mg} /$ week) and levetiracetam, which had been prescribed since her last visit. A fundus examination revealed peripapillary atrophy, but no signs of disc edema in either the left or the right eye. Visual field tests were abnormal (Humphrey field test, VFI was $61 \%$ in right eye and $71 \%$ in left eye). One month later, she was given injections of rituximab.

At the final eye examination 10 months after her first visit, BCVA was $0.8 / 0.65$. A slight improvement of visual test results was observed (Humphrey field test, VFI was $83 \%$ in right eye and $74 \%$ in left eye). As noted previously, color vision was more defective in the left eye ( $35 \mathrm{p}$ ) compared to the right (20 p), although some improvement was seen since the last examination. 


\section{Patient 7}

A 56-year-old woman with a history of diabetes, hypertension, and heart failure developed a gradual decrease in vision in her left eye over a 2-month period prior to her first examination. Two weeks prior to her first visit, she developed left eye pain, radiating upward. She negated headaches, diplopia, or problems with her right eye. She was currently on insulin, antihypertensive medication, and aspirin. The results of the first ophthalmologic examination revealed optic disc edema and RAPD in the left eye. Visual acuity was 0.8 in the right eye and 0.13 in the left eye.

Initial laboratory testing included syphilis serology, Lyme disease (Borrelia antibodies), determination of HGB, electrolyte status, ACE, blood count, CRP, and ESR. The results of all the tests were negative or normal except Lyme disease serology (IgG 239 (pos), IgM 9 (neg)), low ACE (2 U/L), and eosinophilic cells (high).

An MRI performed 1 week later revealed enlargement and enhancement in the left nerve sheath from the eye to the chiasma, in line with the findings seen in OPN (Figures 1 and 2).

At a new consultation 1 month after her first visit, BCVA was unchanged. A visual field test revealed a general constriction of the visual field of the left eye, with equivalent neural capacity of $99 \%$ in his right eye and $33 \%$ in his left eye. Fundus examination showed progression of the left optic disc edema (Figure 3). RAPD was still present. New laboratory tests that included ESR, CRP, ACE, and sarcoidosis revealed nothing abnormal.

One month later, 2 months after her first examination, the left optic disc edema had regressed but an RAPD was

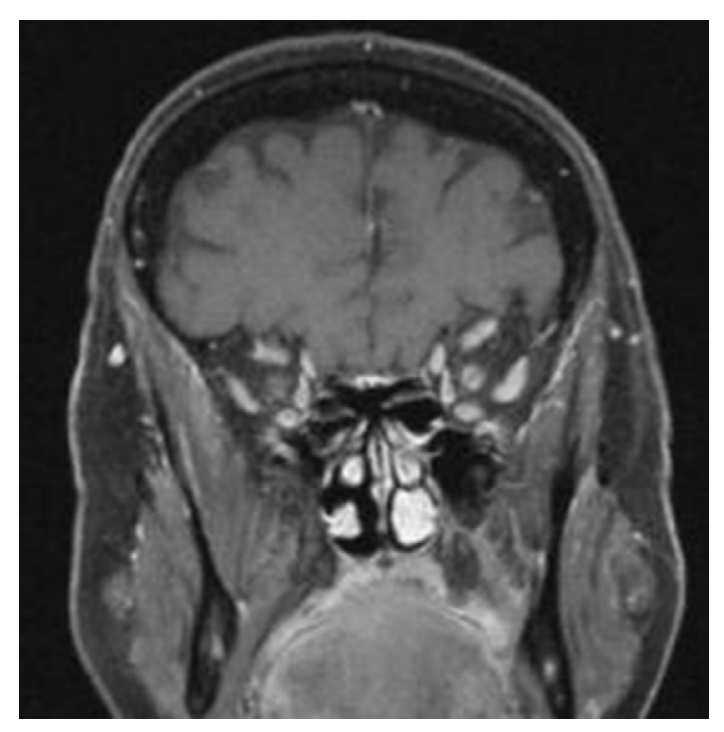

Figure I Coronal post-contrast TI fat-saturated magnetic resonance imaging of Patient 7 demonstrating circumferential enhancement in the left optic nerve sheath.

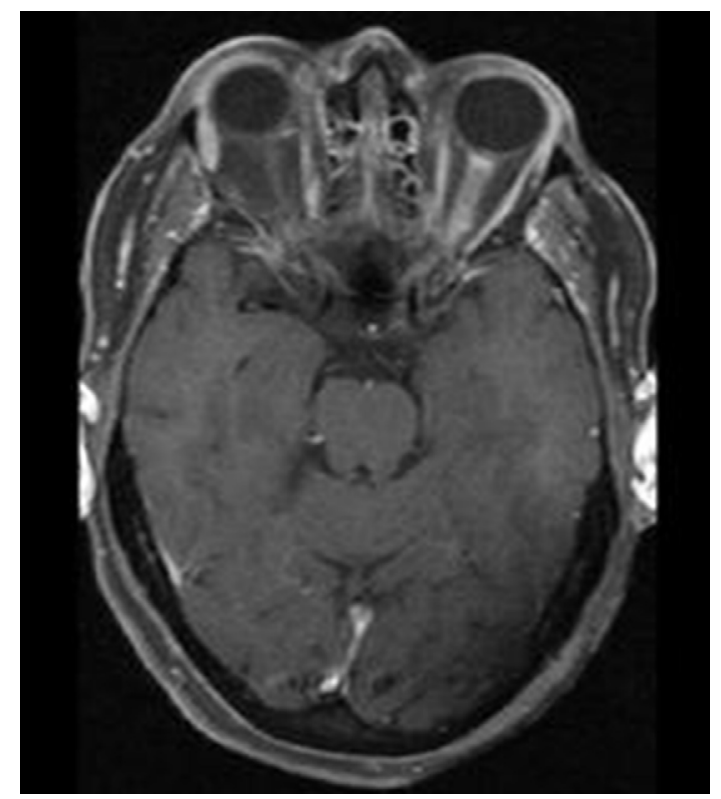

Figure 2 Axial post-contrast TI fat-saturated magnetic resonance imaging of Patient 7 showing enhancement in the left optic nerve sheath from the eye to the chiasma.

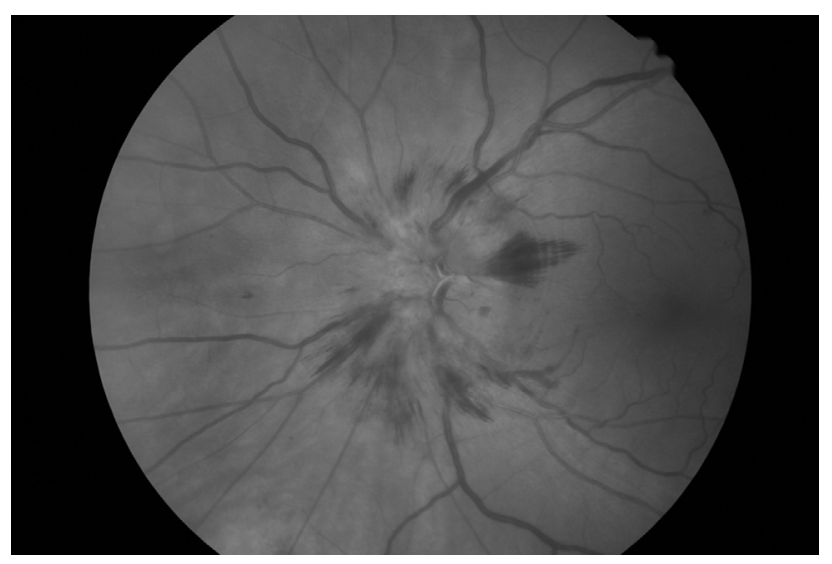

Figure 3 Fundus photo of Patient 7 I month after first examination, showing an optic disc edema.

still present. Visual acuity was unchanged since her last visit. Treatment with prednisone was initiated, $60 \mathrm{mg} /$ day, which was tapered to $25 \mathrm{mg}$ /day over 4 weeks when she was examined again.

At a fourth consultation, 3 months after her first visit, visual acuity was 0.8 in both eyes. A SST color vision test revealed that the patient had more defective color vision in her left eye ( $35 \mathrm{p})$, compared to the right (15 p). The optic disc edema was reduced. The steroid dose was tapered to $5 \mathrm{mg} /$ day during 3 weeks. At this point, the patient suffered her first relapse with dimmed vision in her left eye. Fundus examination 1 week later revealed a pale papilla. BCVA was now 0.8 in her right eye and 0.4 in her left eye. Steroid dose was increased to $30 \mathrm{mg} /$ day and tapered to $20 \mathrm{mg} /$ day until the 
next appointment 2 weeks later. Some days later, the patient was hospitalized with arrhythmia due to hypercalcemia and hyperkalemia. At the new consultation, visual acuity was 0.8 in her right eye and 0.5 in her left eye.

The steroid dose was lowered with $2.5 \mathrm{mg}$ per week. At $10 \mathrm{mg} /$ day, she suffered a second relapse and received intravenous steroids. Her corticosteroid dose was increased to $30 \mathrm{mg} /$ day in addition to azathioprine $(150 \mathrm{mg} /$ day $)$. A fundus examination 3 months later revealed a normal optic disc with no edema. One month later, the steroid dose had been tapered to $17.5 \mathrm{mg}$ /day. BCVA was 0.8 in both eyes. An SST color vision test revealed defective color vision in both the right ( $30 \mathrm{p})$ and the left eye (40 p). The fundus examination showed no sign of optic disc edema.

Four months later, it was suspected that the patient was suffering from sarcoidosis. Liver and spleen biopsies were positive for granulomas.

Four months later, she had a third relapse. She was taking $150 \mathrm{mg}$ azathioprine and no corticosteroids at this time. Both eyes were affected; BCVA was 0.4 in her left eye and 0.65 in her right eye. A fundus examination revealed blurred borders of the optic discs in both eyes.

At a new consultation 2 months later, she remained on azathioprine, $150 \mathrm{mg} /$ day, and was taken off corticosteroids. BCVA was 0.5 in her right eye and 0.65 in her left eye. Fundus examination showed no optic disc edema. A new MRI added support to the OPN diagnosis.

\section{Discussion}

The term OPN is most commonly used to describe a form of orbital inflammatory disease in which an inflammatory process in the optical nerve sheath is present. The cause of OPN often remains unknown. Given the number of associated disorders, OPN is possibly a heterogeneous disease, which has nerve sheath inflammation in common. The present report includes both idiopathic and possible secondary cases, which are summarized in Table 1. Patients $1-5$ seem to have suffered from idiopathic forms of the disease. Patient 6 had a history of SLE and Patient 7 was diagnosed with sarcoidosis. Both SLE and sarcoidosis are inflammatory diseases and plausible underlying causes of OPN.

In the present study, the idiopathic cases typically experienced unilateral visual loss over several days. In contrast, the secondary cases (Patients 6 and 7) displayed a gradual decrease in visual acuity over several weeks.

Four of the seven patients in the present study (Patients $1,4,5$, and 7) complained of ocular pain or pain related to eye movement. This is comparable to a previous case series of idiopathic OPN in which 8 of 14 patients had pain as a presenting feature. ${ }^{2}$ A later case review reported that pain or discomfort was present in 14 out of 19 affected eyes. ${ }^{4}$

Visual field loss with sparing of central visual acuity was reported as a presenting feature in Patients 2, 4, and 6. Visual field tests in Patient 1 were normal. Patients 3, 5, and 7 were not initially evaluated. However, a visual field test of Patient 3 was performed 8 months after initial symptoms began and revealed a lower arcuate scotoma in the right eye. Patient 5 had a visual field test 2 months after initial symptoms began that was normal except for a small isolated central scotoma with a slightly elevated threshold in his left eye. A visual field test in Patient 7 was performed 1 month after her first examination and showed reduced neural capacity in her left eye and normal conditions in her right eye.

Initially, Patients 2, 5, and 7 had optic disc edemas, Patient 6 had peripapillary atrophy, and the remaining patients had normal optic discs at their first examinations. During subsequent examinations, Patient 3 had a pale and slightly atrophic optic disc. This is comparable with previous studies of OPN, which reported optic disc edemas in 10 of 15 affected eyes, ${ }^{2}$ and 10 out of 18 patients, ${ }^{4}$ respectively.

One important difference between OPN and ON is that the latter is coupled to an increased risk of developing MS. As expected in OPN, none of the patients included in this study displayed abnormalities typical of MS, ie, demyelinated areas, at the MRI scans.

Age is another factor where OPN typically differs from ON. The mean age of patients at onset of OPN is generally older than age at onset of $\mathrm{ON}$. The average age in the present study was 55 years (range 26-64 years). A case series from 2001 consisting of 14 patients with idiopathic OPN had a mean age at presentation of 41 years (range $24-60$ ). ${ }^{2}$ A more recent review of case reports from 2016 reported a mean age of 57 (range 15-85) in patients with idiopathic OPN. ${ }^{4}$

Five of the patients received corticosteroid treatment, which is important in treatment of OPN, if not patients are to suffer continued loss of vision. Prognosis in OPN with regard to visual outcome is usually very good. It has been reported that outcome is dependent on time between onset of symptoms and initiation of corticosteroid treatment. ${ }^{2}$ In contrast, corticosteroid treatment is of lesser value in patients with $\mathrm{ON}$, which often are managed expectantly or with shorter regimens. The high-dose corticosteroid treatment used to treat OPN may be associated with relapse of ON and should consequently be avoided if diagnosis is uncertain. ${ }^{2}$

Of the patients with idiopathic OPN, Patients 2 and 3 received a starting dose of $60 \mathrm{mg}$ prednisone per day. Patient 
4 received intravenous methylprednisone ( $1 \mathrm{~g} /$ day) for 5 days prior to oral prednisone $(60 \mathrm{mg} /$ day $)$. Treatment was delayed for 2 weeks in Patient 2 and almost 3 months in Patient 3. Corticosteroid treatment was tapered over several months. Patient 4 had a faster taper of corticosteroids and suffered a relapse after complete treatment. BCVA was improved in all patients between the first and the last examination (Table 1).

Patients 6 and 7 had underlying inflammatory disorders. Patient 6 received intravenous methylprednisone, followed by prednisone and cyclophosphamide on account of her SLE. Because of heart arrhythmia, treatment was quickly restricted to a low dose of prednisone ( $5 \mathrm{mg}$ /day). BCVA did not improve between the first and the last examination. Patient 7 was treated with $60 \mathrm{mg}$ of prednisone per day. Treatment was delayed for 4 months, and the patient experienced three relapses as the dose was lowered, each occurring at a daily dose of around $10 \mathrm{mg}$ prednisone. BCVA was improved between the first and the last visit (Table 1).

Guided by the results of ocular and systemic examinations, patients with suspected OPN should be investigated for underlying systemic disorders with appropriate serum tests and radiological examinations.

OPN is a disorder that in many respects mimics that of ON. It is possible that misdiagnosed ONs, which result in seemingly benign cases of MS, are in fact OPN. The findings of this study add support to the notion that OPN should be considered when $\mathrm{ON}$ is suspected, in patients of older age and with sparing of central vision. In patients with suspected OPN, MRI of the brain and orbita is essential for correct diagnosis. Moreover, this study suggests that a high-dose course of corticosteroids that is tapered slowly is important in the treatment of OPN in severely affected patients.

\section{Disclosure}

The authors report no conflicts of interest in this work.

\section{References}

1. Kennerdell JS, Dresner SC. The nonspecific orbital inflammatory syndromes. Surv Ophthalmol. 1984;29(2):93-103.

2. Purvin V, Kawasaki A, Jacobson DM. Optic perineuritis: clinical and radiographic features. Arch Ophthalmol. 2001;119(9):1299-1306.

3. Purvin V, Kawasaki A. Optic perineuritis secondary to Wegener's granulomatosis. Clin Exp Ophthalmol. 2009;37(7):712-717.

4. Hickman SJ. Optic perineuritis. Curr Neurol Neurosci Rep. 2016;16(2):16.

5. Toshniwal P. Optic perineuritis with secondary syphilis. J Clin Neuroophthalmol. 1987;7(1):6-10.

6. O'Connell K, Marnane M, McGuigan C. Bilateral ocular perineuritis as the presenting feature of acute syphilis infection. J Neurol. 2012;259(1):191-192.

7. Delaney P. Neurologic manifestations in sarcoidosis: review of the literature, with a report of 23 cases. Ann Int Med. 1977;87(3):336-345.

8. Meehan K, Rodman J. Ocular perineuritis secondary to neurosyphilis. Optom Vis Sci. 2010;87(10):E790-E796.

9. McClelland C, Zaveri M, Walsh R, Fleisher J, Galetta S. Optic perineuritis as the presenting feature of Crohn disease. J Neuroophthalmol. 2012;32(4):345-347.

10. Tatsugawa M, Noma H, Mimura T, Funatsu H. High-dose steroid therapy for idiopathic optic perineuritis: a case series. J Med Case Rep. 2010;4:404

11. Tung C, Hashemi N, Lee AG. Spontaneous resolution of optic perineuritis. J Neuroophthalmol. 2013;33(1):93-95.

12. Hoorbakht H, Bagherkashi F. Optic neuritis, its differential diagnosis and management. Open Ophthalmol J. 2012;6:65-72.

13. Fay AM, Kane SA, Kazim M, Millar WS, Odel JG. Magnetic resonance imaging of optic perineuritis. J Neuroophthalmol. 1997;17(4):247-249.

14. Tien RD, Hesselink JR, Szumowski J. MR fat suppression combined with Gd-DTPA enhancement in optic neuritis and perineuritis. J Comput Assist Tomogr. 1991;15(2):223-227.
International Medical Case Reports Journal

\section{Publish your work in this journal}

The International Medical Case Reports Journal is an international, peer-reviewed open-access journal publishing original case reports from all medical specialties. Previously unpublished medical posters are also accepted relating to any area of clinical or preclinical science. Submissions should not normally exceed 2,000 words or
Dovepress

4 published pages including figures, diagrams and references. The manuscript management system is completely online and includes a very quick and fair peer-review system, which is all easy to use. Visit $\mathrm{http}: / /$ www.dovepress.com/testimonials.php to read real quotes from published authors. 